\title{
Influence of a uniform magnetic field on dynamical chiral symmetry breaking in $\mathrm{QED}_{3}$
}

\author{
Jian-Feng Li ${ }^{1,2}$, Hong-Tao Feng ${ }^{3}$, Yu Jiang ${ }^{4}$, Wei-Min Sun ${ }^{2,5}$ and Hong-Shi Zong ${ }^{2,5}$ \\ 1 College of Mathematics and Physics, \\ Nantong University, Nantong 22600\%, China \\ 2 Department of Physics, Nanjing University, Nanjing 210093, China \\ 3 Department of Physics, Southeast University, Nanjing 211189, China \\ 4 Center for Statistical and Theoretical Condensed Matter Physics, \\ Zhejiang Normal University, Jinhua 321004, China and \\ 5 Joint Center for Particle, Nuclear Physics and Cosmology, Nanjing 210093, China
}

\begin{abstract}
We study dynamical chiral symmetry breaking (DCSB) in an effective $\mathrm{QED}_{3}$ theory of $d$-wave high temperature cuprate superconductors under a uniform magnetic field. At zero temperature, the external magnetic field induces a mixed state by generating vortices in the condensate of charged holons. The growing magnetic field suppresses the superfluid density and thus reduces the gauge field mass which is opened via the Anderson-Higgs mechanism. By numerically solving the Dyson-Schwinger gap equation, we show that the massless fermions acquires a dynamical gap through DCSB mechanism when the magnetic field strength $H$ is above a critical value $H_{c}$ and the fermion flavors $N$ is below a critical value $N_{c}$. Further, it is found that both $N_{c}$ and the dynamical fermion gap increase as the magnetic field $H$ grows. It is expected that our result can be tested in phenomena in high temperature cuprate superconductors.
\end{abstract}

Key-words: $\mathrm{QED}_{3}$; dynamical chiral symmetry breaking; gap equation; $d$-wave superconductor

E-mail: zonghs@chenwang.nju.edu.cn. $\quad$ PACS Numbers: 11.10.Kk, 11.15.Tk, 11.30.Qc 
Analogous to Quantum Chromodynamics (QCD), Quantum Electrodynamics in $(2+1)$ spacetime dimensions $\left(\mathrm{QED}_{3}\right)$ has some interesting features, such as dynamical chiral symmetry breaking (DCSB) in the massless fermion limit and confinement [1 9$]$. Therefore $\mathrm{QED}_{3}$ can be regarded as a useful model by which one can gain important insights into the aspects of QCD. In addition, $\mathrm{QED}_{3}$ and its variants also have proved to be the effective field theories for some two-dimensional condensed matter systems, including high temperature superconductors [10 18] and graphene [19 27]. Because of these features, $\mathrm{QED}_{3}$ has been extensively studied in recent years.

DCSB occurs when the massless fermions appearing in a Lagrangian that respects chiral symmetry acquire a nonzero mass through nonperturbative effects at low energy. In a four-fermion interaction model, Nambu and Jona-Lasinio [28] first transplanted the fermion pairing idea in the BCS theory of superconductivity into particle physics to generate a fermion mass without assuming a Yukawa type coupling between fermion and Higgs scalar field (using Yukawa type coupling). In Refs. [3, 4] Appelquist et al. first studied DCSB in massless $\mathrm{QED}_{3}$ with $N$ fermion flavors at zero temperature by solving the Dyson-Schwinger equations (DSEs) for fermion self-energy in the lowest-order of $1 / N$ expansion, and found that a fermion mass was dynamically generated when $N$ is less than a critical number $N_{c}=$ $32 / \pi^{2}$. Later, Nash further considered higher order corrections, and showed that the critical number of fermion flavor still exists and $N_{c}=\frac{128}{3 \pi^{2}}$ [5]. At finite temperature, Aitchison et al. used an approximate treatment of DSEs for the fermion self-energy in the $1 / N$ expansion [29] and found that $N_{c}$ is temperature-dependent and chiral symmetry is restored above a certain critical temperature. Further analogy with QCD at finite temperature and chemical potential has been explored [30 33]. The methods described there could be employed to extend the study of $\mathrm{QED}_{3}$ at nonzero temperature and chemical potential and explore whether and under which condition chiral symmetry restoration and deconfinement are coincident. Recently, the authors in Ref. [34] studied the impact of a uniform magnetic field on explicit chiral symmetry breaking in $\mathrm{QED}_{3}$.

Besides its interests in high energy physics, $\mathrm{QED}_{3}$ also turned out to be an effective low-energy theory of high temperature cuprate superconductors. In the past twenty years, it has been extensively applied to understand some unusual physics of high temperature superconductors [13]. In two early papers [35, 36], it was shown that the half-filled state on the $\mathrm{CuO}_{2}$ plane of undoped cuprates can be mapped to a (2+1)-dimensional continuum field 
theory composed of massless Dirac fermions, called spinons, and U(1) gauge field, which is exactly $\mathrm{QED}_{3}$. In this effective theory, the physical fermion flavor is $N=2<N_{c}$, so DCSB can take place at zero temperature. Physically, DCSB corresponds to the formation of long-range antiferromagnetic (AFM) order [37, 38]. At finite doping concentration, the simple $\mathrm{QED}_{3}$ of Dirac fermions is not sufficient to model the dynamics of doped holes. To overcome this difficulty, Lee and Wen developed an SU(2) gauge field theory to describe the underdoped cuprates [13]. In the so-called staggered flux phase, two components of the $\mathrm{SU}(2)$ gauge field are gapped and thus can be neglected, leaving only one massless component of gauge field [13]. However, in underdoped cuprates, there appear additional scalar boson excitations, called holons, which describes the dynamics of doped holes. Therefore, the effective continuum theory of underdoped cuprates is an extended $\mathrm{QED}_{3}$ theory of massless spinons and scalar holons [37, 38]. The spinons and holons both interact with the gauge field strongly, but there is no direct coupling between them. This theory can exhibit a lot of interesting features, and has been investigated extensively in the past several years [13, 35 -39].

Within this effective theory, superconductivity is achieved by the condensation of holons at low temperature. In the superconducting (SC) state, the gauge field acquires a finite mass gap through the famous Anderson-Higgs ( $\mathrm{AH}$ ) mechanism. As shown in Ref. [40], this gauge field mass weakens the gauge interaction and thus rapidly suppresses DCSB. If we use DCSB to represent AFM order and use gauge field mass to represent SC order, then the competition between AFM order and SC order can be understood by the competition between DCSB and AH mechanism [40].

Now suppose we introduce an external magnetic field into the SC state, then a mixed state, or called vortex state, emerges since high temperature superconductors are known to be extreme type-II superconductors. The most important influence of external magnetic field is to suppress the superfluid density of the SC state. From a field-theoretic point of view, this implies the suppression of the gauge field mass, which is proportional to the superfluid density. Since the gauge field mass can have important influence on DCSB, it is interesting to study the fate of DCSB when the strength of external magnetic field is varied. The main purpose of this paper is to study the impact of the external magnetic field on the critical fermion flavor $N_{c}$.

In this paper, we first employ the DSEs method to construct the fermion gap equation 
at zero temperature after including the effect of an external uniform magnetic field. Then we solve the gap equation to study the influence of external magnetic field on DCSB in the mixed state of a d-wave cuprate superconductor. The main result of our paper is that both the critical fermion flavor $N_{c}$ and the gap increase with growing magnetic field.

It is well known that $\mathrm{QED}_{3}$ is super-renormalizable and has an intrinsic mass scale given by the coupling constant $\alpha=\frac{N e^{2}}{8}$. In Euclidean space, the Lagrangian of massless $\mathrm{QED}_{3}$ with $N$ fermion flavors reads

$$
\mathcal{L}=\sum_{i=1}^{N} \bar{\psi}_{i}(\not \partial+\mathrm{i} e \not h) \psi_{i}+\frac{1}{4} F_{\rho \nu}^{2}+\frac{1}{2 \xi}\left(\partial_{\rho} a_{\rho}\right)^{2}
$$

where the $4 \times 1$ spinor $\psi_{i}$ represents the fermion field, $i=1, \cdots, N$ are the flavor indices, and $\xi$ is the gauge parameter. Here, the massless Dirac fermions are not the electron-like excitations, but are actually the gapless spinons excited from the nodes of $d$-wave energy gap. These fermions have spin $1 / 2$ but are electrically neutral. The gauge field $a_{\mu}$ is also not the usual vector potential of electromagnetic field. Instead, it emerges as the consequence of strong correlation between electrons on the $\mathrm{CuO}_{2}$ planes of high- $T_{c}$ superconductors. This effective field theory can be derived from the microscopic $t-J$ model of high- $T_{c}$ superconductors with the help of slave-boson technique [13].

The DSEs for the fermion propagator $G(p)$ at zero temperature reads

$$
G^{-1}(p)=i \gamma \cdot p+e^{2} \int \frac{d^{3} k}{(2 \pi)^{3}} \gamma_{\rho} G(k) \Gamma_{\nu}(p, k) D_{\rho \nu}(q),
$$

where $D_{\rho \nu}$ is the propagator of the $a_{\mu}$ field and $q=p-k$. For simplicity, in this paper we adopt the rainbow approximation, i.e., we approximate the full vertex function $\Gamma_{\nu}(\mathrm{p}, \mathrm{k})$ with the bare vertex $\gamma_{\nu}$ and we neglect the wave function renormalisation (this is a commonly employed approximation in the study of fermion propagator of $\mathrm{QED}_{3}$ at finite temperature [19, 41, 42]). Similar with the usual DSE study, in the following we will work in Landau gauge.

At finite temperature $T$ the DSEs for the fermion propagator is given by

$$
G^{-1}(p)=G_{0}(p)-\frac{e^{2}}{\beta} \sum_{n=-\infty}^{\infty} \int \frac{d^{2} k}{(2 \pi)^{2}} \gamma^{\mu} G\left(k_{0}, K, \beta\right) \Delta_{\mu \nu}\left(q_{0}, Q, \beta\right) \gamma^{\nu},
$$

where

$$
G(p)=\frac{1}{i p_{0} \gamma_{0}-v_{F} p_{1} \gamma_{1}-v_{\Delta} p_{2} \gamma_{2}-m\left(p^{2}\right)}
$$


is the fermion propagator at finite temperature and $m(p)$ represents the Dirac fermion mass. Here $v_{F}$ is the Fermi velocity, $v_{\Delta}$ is the gap velocity. Since the velocity anisotropy is unimportant to the critical behavior [18], we can set $v_{F}=v_{\Delta}=1$, and

$$
\begin{array}{r}
p=\left(p_{0}, \mathbf{p}\right), P=|\mathbf{p}|, p_{0}=(2 m+1) \frac{\pi}{\beta}, \\
k=\left(k_{0}, \mathbf{k}\right), K=|\mathbf{k}|, k_{0}=(2 n+1) \frac{\pi}{\beta}, \\
q=\left(q_{0}, \mathbf{q}\right), Q=|\mathbf{q}|=|\mathbf{p}-\mathbf{k}|, q_{0}=2(m-n) \frac{\pi}{\beta} .
\end{array}
$$

Under the above approximation the inverse of the fermion propagator can be written as

$$
G^{-1}(p)=i \gamma \cdot p+m\left(p^{2}\right)
$$

Taking the trace of both sides of Eq. (3) yields a closed integral equation for m(p) [29, 41, 42]:

$$
m\left(p^{2}\right)=\frac{\alpha}{N \beta} \sum_{n=-\infty}^{\infty} \int \frac{d^{2} k}{(2 \pi)^{2}} \Delta\left(q_{0}, Q, \beta\right) \frac{m\left(k^{2}\right)}{k^{2}+m^{2}\left(k^{2}\right)}
$$

with

$$
\Delta\left(q_{0}, Q, \beta\right)=\frac{1}{8} \operatorname{Tr}\left[\gamma_{\mu} \Delta_{\mu \nu}\left(q_{0}, Q, \beta\right) \gamma_{\nu}\right]
$$

where

$$
\Delta_{\mu \nu}\left(q_{0}, Q, \beta\right)=\frac{\delta_{\mu 3} \delta_{\nu 3}}{Q^{2}+\Pi_{0}(Q, \beta)+m_{a}^{2}}
$$

and

$$
\Pi_{0}(Q, \beta)=\frac{\alpha}{8 \beta}\left[Q \beta+\frac{16 \ln 2}{\pi} \exp \left(-\frac{\pi \beta}{16 \ln 2}\right)\right] .
$$

When the photon acquires a mass $m_{a}$ via the Anderson-Higgs mechanism, the massless photon propagator is replaced by the massive photon propagator (9). So we have

$$
m\left(p^{2}\right)=\frac{\alpha}{8 N \beta} \sum_{n=-\infty}^{\infty} \int \frac{d^{2} k}{(2 \pi)^{2}} \frac{m\left(k^{2}\right)}{\left[K^{2}+m^{2}\left(k^{2}\right)+\varpi_{n}^{2}\right]\left[Q^{2}+\Pi_{0}(\beta, Q)+m_{a}^{2}\right]} .
$$

The summation over infinite Matsubara frequencies can be done analytically:

$$
\begin{aligned}
& \sum_{n=-\infty}^{\infty} \frac{1}{\varpi_{n}^{2}+K^{2}+m^{2}\left(k^{2}\right)} \\
= & \frac{1}{2 \sqrt{K^{2}+m^{2}\left(k^{2}\right)}} \sum_{n=-\infty}^{\infty}\left[\frac{1}{i \varpi_{n}+\sqrt{K^{2}+m^{2}\left(k^{2}\right)}}-\frac{1}{i \varpi_{n}-\sqrt{K^{2}+m^{2}\left(k^{2}\right)}}\right] \\
= & \frac{\beta}{2 \sqrt{K^{2}+m^{2}\left(k^{2}\right)}} \tanh \left[\frac{\beta}{2} \sqrt{K^{2}+m^{2}\left(k^{2}\right)}\right] .
\end{aligned}
$$


Finally we obtain the temperature- and momentum-dependent gap function

$$
m\left(p^{2}\right)=\frac{\alpha}{8 N \pi^{2}} \int d^{2} K \frac{m\left(k^{2}\right)}{\sqrt{K^{2}+m^{2}\left(k^{2}\right)}} \frac{\tanh \left[\frac{\beta}{2} \sqrt{K^{2}+m^{2}\left(k^{2}\right)}\right]}{Q^{2}+\Pi_{0}(\beta, Q)+m_{a}^{2}} .
$$

Here we note that in the limit of zero temperature $\beta \rightarrow \infty$, we have

$$
\Pi_{0}(Q, \beta) \rightarrow \frac{\alpha Q}{8}
$$

and the gap equation further simplifies into

$$
m\left(P^{2}\right)=\frac{\alpha}{8 N \pi^{2}} \int d^{2} K \frac{m\left(K^{2}\right)}{\sqrt{K^{2}+m^{2}\left(K^{2}\right)}} \frac{1}{Q^{2}+\frac{\alpha Q}{8}+m_{a}^{2}} .
$$

In order to see how the gauge field acquire a mass $m_{a}$, we now introduce the additional interaction term between gauge field and scalar boson field

$$
\mathcal{L}_{h}=\phi^{*}\left(\partial_{0}+i a_{0}\right) \phi-\frac{1}{2 m_{h}} \phi^{*}(\partial+i \mathbf{a})^{2} \phi+\frac{u}{2} \phi^{2}+\frac{\beta}{4} \phi^{4},
$$

which is so-called Abelian Higgs model. The scalar field $\phi$ represents the bosonic holons, which has spin-0 and carry charge $-e$ [13]. This Lagrangian describes the motion of the charge degrees of freedom of electrons on the $\mathrm{CuO}_{2}$ planes of underdoped cuprate superconductors [13]. Unfortunately, there is currently not a clear understanding of the dynamics of holons. In previous treatments of this model, the holon sector was simply neglected [37-39] in the analysis of the interaction between Dirac fermions and gauge field. In this paper, we believe that the most prominent effect of this additional interaction is to generated a finite mass for the gauge field in the SC state. When $u>0$, the system stays in the normal state and the vacuum expectation value of boson field $\langle\phi\rangle=0$, so the Lagrangian respects the local gauge symmetry. When $u<0$, the system enters the SC state and the boson field develops a finite expectation value $\langle\phi\rangle \neq 0$, then the local gauge symmetry is spontaneously broken. According to the Anderson-Higgs mechanism, the gauge field acquires a finite mass $m_{a}$ after absorbing the massless Goldstone boson generated due to spontaneous breaking of continuous gauge symmetry. The finite gauge field mass is able to characterize the achievement of superconductivity. Actually, this mass is proportional to the superfluid density of the SC state, $m_{a} \propto\langle\phi\rangle$.

The external magnetic field turns the uniform SC state to a mixed state, where the superfluid density and therefore the gauge field mass decreases with growing field strength 
$H$. If we can get a relationship between gauge field mass $m_{a}$ and field strength $H$, we will be able to study the impact of magnetic field on DCSB. Since the holon dynamics is poorly understood, we will use phenomenological arguments.

As mentioned above, DCSB can describe the AFM order and the gauge boson mass can describe the SC order [11, 40]. Based on these correspondences, we are able to give a qualitatively physical description of the competition between AFM order and SC order in the mixed state of high- $T_{c}$ superconductors. In the absence of external magnetic field, the system stays in a uniform SC state at zero temperature, with a superfluid density $\Lambda_{s}$. As an external magnetic field is introduced, the phase coherence among holons is disputed and there appear single vortices inside which superconductivity is destroyed. In this mixed state, the superfluid density becomes inhomogeneous and takes different value at different positions. However, after averaging over the vortices, it is still possible to obtain a homogeneous superfluid density $\Lambda_{s}(H)$, which is a function of magnetic field. On physical grounds, it is easy to suppose that $\Lambda_{s}(H)$ is a decreasing function of field strength $H$. The gauge boson mass in the mixed state is proportional to the superfluid density, so $m_{a}=a \Lambda_{s}(H)$, where $a$ is a dimensionless constant which depends on the doping concentration. Now we have a clear picture: as magnetic field $H$ grows, the superfluid density $\Lambda_{s}(H)$ decreases, and hence the gauge boson mass $m_{a}$ decreases, which will raise the critical fermion flavor $N_{c}$ and may finally induce DCSB in the mixed state. Once a finite fermion mass is dynamically generated, its magnitude $m\left(p^{2}\right)$ will be an increasing function of magnetic field $H$. In the following we will verify this qualitative argument by numerically solving the gap equations in the presence of an external magnetic field.

To study the gap equation, the superfluid density $\Lambda_{s}(H)$ can be obtained by averaging over the vortices. In the study of mixed state, Volovik [43] at first proposed a semiclassical approach and showed that the density of states goes as $\sqrt{H}$ at low temperatures, which has been observed by experiments [44]. A more complicated computation of superfluid density $\Lambda_{s}(H)$ within the semi-classical approximation has already been performed by Sharapov et al. [45]. The superfluid stiffness is given by

$$
\Lambda_{s}^{i j}(T, H)=\tau^{i j}-\Lambda_{n}^{i j}(T, H),
$$

where $\tau_{i j}$ is the diamagnetic tensor and $\Lambda_{n}$ represents the normal fluid density divided by the carrier mass. After taking some approximations and performing some complicated 
calculations, Sharapov et al. obtained [45]

$$
\Lambda_{n}(H)=\frac{v_{F}}{\pi v_{\Delta}} \frac{E_{H}^{2}}{\sqrt{E_{H}^{2}+\Delta_{d_{x y}}^{2}}} .
$$

When the superconducting gap $\Delta_{d_{x y}}(H) \ll \sqrt{H}$, we can neglect the $\Delta_{d_{x y}}$ and $\Lambda_{n}(H)$ still has the behavior $\sim \sqrt{H}$. The superfluid density then becomes

$$
\Lambda_{s}(H)=\tau-\frac{v_{F}}{\pi v_{\Delta}} E_{H}
$$

Here, $\tau$ is the superfluid density at zero temperature in the absence of magnetic field and the ratio between $v_{F}$ and $v_{\Delta}$ appears as a coefficient. When $v_{F}$ and $v_{\Delta}$ multiply particle momenta, the anisotropy is irrelevant, but the anisotropy is very important in this expression. In the following discussions, as in Ref. [45], we simply set the ratio $v_{F} / v_{\Delta}=30$, and at zero temperature the superfluid density is taken to be $\tau=1500 \mathrm{~K}$ and the energy scale $E_{H}[K]=38 \mathrm{KT}^{-1 / 2} \sqrt{H[T]}$. Since the gauge boson $m_{a}$ is proportional to superfluid density, we use the ansatz that $m_{a}=a \Lambda_{s}(H)$, where parameter $a$ is a free variable.

After obtaining the effective gauge boson propagator (15), we can now obtain the fermion gap equation in the presence of an external magnetic field

$$
m\left(P^{2}\right)=\frac{\alpha}{8 N \pi^{2}} \int d^{2} K \frac{m\left(K^{2}\right)}{\sqrt{K^{2}+m^{2}\left(K^{2}\right)}} \frac{1}{Q^{2}+\frac{\alpha Q}{8}+\left[a \Lambda_{s}(H)\right]^{2}} .
$$

The iteration algorithm can be employed to numerically solve this equation. In our numerical computations, we set $\alpha=1$ so that each quantity with mass dimension is re-scaled and becomes dimensionless. In the real numerical calculations, we adopt an ultraviolet cutoff $\Lambda=10^{5}$, which is large enough to ensure that the calculated results are stable with respect to $\Lambda$. We consider $a=0.07,0.1,0.15$. For each parameter $a$, the relation between the critical fermion flavor $N_{c}$ and the magnetic field $H$ is shown in Fig. 1. The critical number of fermion flavor $N_{c}$ separates the chiral symmetric phase $\left(m=0\right.$ for $\left.N>N_{c}\right)$ from the DCSB phase ( $m>0$ for $N<N_{c}$ ). From Fig.1 it can be seen that for fixed value of the parameter $a, N_{c}$ increases as the magnetic field $H$ grows. For a fixed magnetic field $H$, the smaller is the parameter $a$, the easier is it to generate an energy gap by the mechanism of DCSB. For the physical flavor $N=2$, when the magnetic field $H$ is large enough, a finite mass gap is generated in the mixed state. It is obvious that the dynamical fermion mass will affect all observable physical quantities. It is expected that our result can be tested in phenomena in high temperature cuprate superconductors. 
We now take the physical fermion flavor $N=2$, and numerically solve the gap equation by iteration method for the specific case $a=0.07$. The momentum dependence of the fermion gap is plotted in Fig. 2. From Fig. 2, it can be seen that the dynamically generated mass $m\left(p^{2}\right)$ is almost constant for small momenta, but decreases down to nearly zero at large momenta for a fixed magnetic field. This is easy to understand because the $\mathrm{QED}_{3}$ theory is asymptotically free. Another important feature is that fermion gap $m(H)$ is an increasing function of the external magnetic field $H$. Apparently, the DCSB that is suppressed by finite gauge boson mass is restored when the magnetic field $H$ becomes sufficiently large. When the fermion gap becomes larger with magnetic field, the thermal conductivity $\kappa \propto \frac{\Gamma_{0}^{2}}{m^{2}(H)+\Gamma_{0}^{2}}$, where $\Gamma_{0}$ is the disorder scattering rate, becomes smaller. There will be a thermal metal-toinsulator transition with growing magnetic field in the limit of $m(H) \gg \Gamma_{0}$. These results qualitatively agree with the conclusion given by Jiang et al. in [46], where the authors introduced a two-dimensional Coulomb interaction between Dirac fermions to generate an excitonic gap.

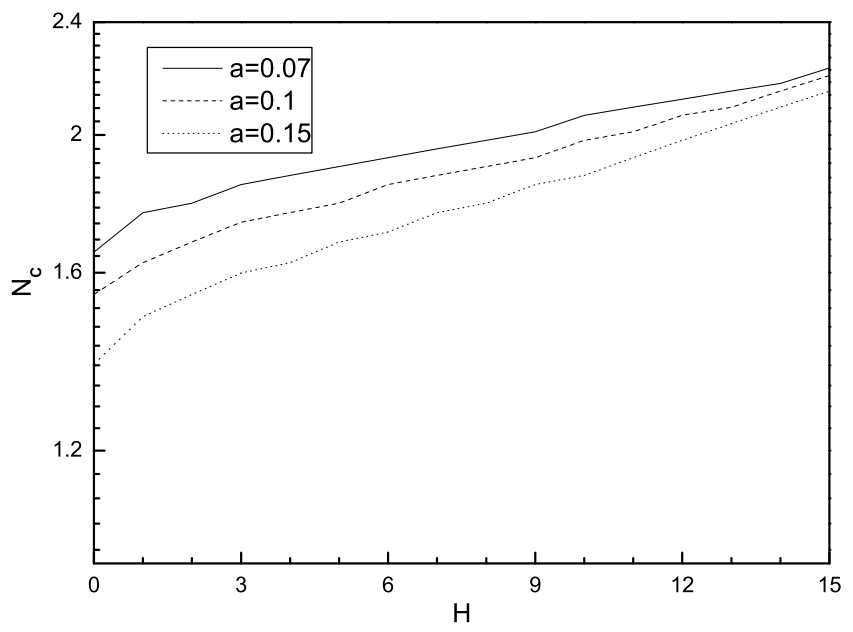

FIG. 1. The dependence of the critical number of fermion flavors $N_{c}$ on the magnetic field for several values of $a$

We note that $\mathrm{QED}_{3}$ in a uniform magnetic field was studied previously in several papers [47 50]. A magnetic catalyst phenomenon was found there, which states that DCSB can be significantly catalyzed by an external magnetic field. In the present paper, this effect is not considered. This is mainly for two reasons. First, the gauge field in Eqs. (1) and (16) 


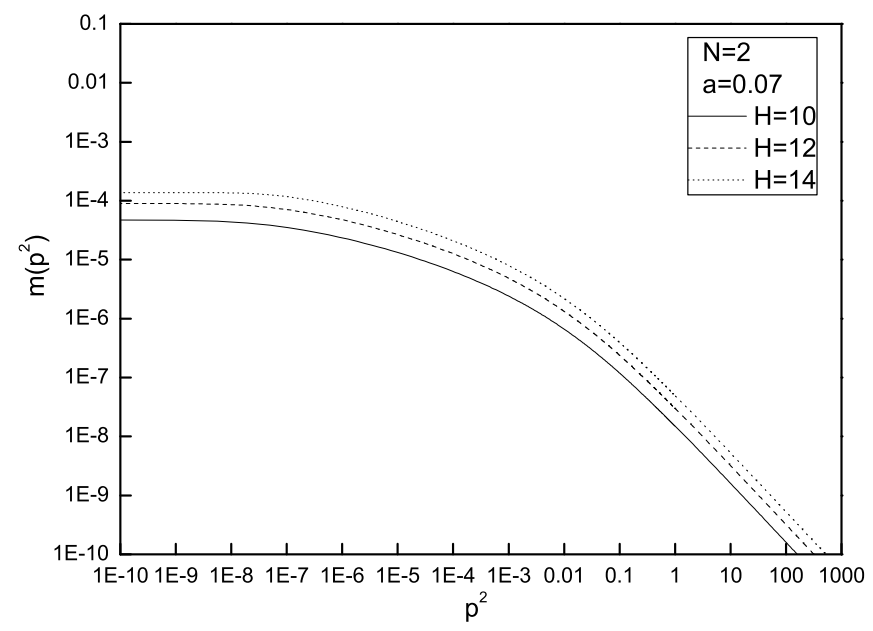

FIG. 2. Scaled mass gap versus scaled momentum at various magnetic field for $N=2$ and $a=0.07$

denoted by $a_{\mu}$, is not the electromagnetic gauge field, but some new internal gauge field. In this sense the fermion does carry a gauge charge which is not the conventional electronic charge. Moreover, unlike the ordinary electrons that carry both spin and electric charge, the Dirac fermions appearing in our model are neutral in electric charge, so they are not directly coupled to the external magnetic field via the minimal coupling principle. Second, a crucial assumption of the magnetic catalyst phenomenon is that the external magnetic field induces discrete Landau levels of the Dirac fermions [47, 49, 50]. However, it was found that there are no Landau levels in the mixed state of high temperature superconductor [51, 52].

To summarize, there is a long history to solve the Dyson-Schwinger gap equation in $\mathrm{QED}_{3}$. It is interesting to apply it to some two-dimensional condensed matter systems. In this paper we apply the analysis to chiral symmetry breaking in high temperature cuprate superconductors under a uniform magnetic field. It is shown that chiral symmetry breaking takes place when the magnetic field is above a critical value $H_{c}$ and the fermion flavors is below a critical value $N_{c}$. Though the well-known magnetic catalysis does not occur in this model, the magnetic field leads to chiral symmetry breaking indirectly by suppressing the gauge boson mass. It is expected that our result can be tested in phenomena in high temperature cuprate superconductors.

\section{Acknowledgement}


We thank G.Z.Liu for helpful correspondence. This work was supported in part by the National Natural Science Foundation of China (under Grant Nos 10175033, 10475057, 10575050), the Research Fund for the Doctoral Program of Higher Education (under Grant No 200802840009) and a project funded by the Priority Academic Program Development of Jiangsu Higher Education Institution.

[1] J.M. Cornwall, Phys. Rev. D 22, 1452 (1980).

[2] R.D. Pisarski, Phys. Rev. D 29, 2423 (1984).

[3] T.W. Appelquist, M. Bowick, D. Karabali and L.C.R. Wijewardhana, Phys.Rev. D 33, 3704 (1986).

[4] T. Appelquist, D. Nash and L.C.R. Wijewardhana, Phys. Rev. Lett. 60, 2575 (1988).

[5] D. Nash,Phys. Rev. Lett. 62, 3024 (1989).

[6] P. Maris, Phys. Rev. D 52, 6087 (1995).

[7] P. Maris, Phys. Rev. D 54, 4049 (1996).

[8] C.J. Burden, J. Praschifka and C.D. Roberts, Phys. Rev. D 46, 2695 (1992).

[9] G. Grignani, G. Semenoff and P. Sodano, Phys. Rev. D 53, 7157 (1996).

[10] X.G. Wen and A. Zee, Phys. Rev. Lett. 69, 953 (1992).

[11] W. Rantner and X.G.Wen, Phys. Rev. Lett. 86, 3871 (2001).

[12] X.G. Wen and P.A. Lee, Phys. Rev. Lett. 76, 503 (1996).

[13] P.A. Lee, N. Nagaosa and X.G. Wen, Rev. Mod. Phys. 78, 17 (2006).

[14] A. Bashir, A. Raya, I.C. Cloet and C.D. Roberts, Phys. Rev. C 78, 055201 (2008).

[15] M. Franz and Z. Tesanovic, Phys. Rev. Lett. 87, 257003 (2001).

[16] I.F. Herbut, Phys. Rev. Lett. 88, 047006 (2002).

[17] I.F. Herbut, Phys. Rev. B 66, 094504 (2002).

[18] D.J. Lee and I.F. Herbut, Phys. Rev. B 66, 094512 (2002).

[19] D.V. Khveshchenko, Phys. Rev. Lett. 87, 246802 (2001).

[20] D.V. Khveshchenko and H. Leal, Nucl. Phys. B 687, 323 (2004).

[21] E.V. Gorbar, V.P. Gusynin, V.A. Miransky and I.A. Shovkovy, Phys. Rev. B 66, 045108 (2002).

[22] V.P. Gusynin and S.G. Sharapov, Phys. Rev. lett. 95 146801(2005). 
[23] V.P. Gusynin, S.G. Sharapov and J.P. Carbotte, Phys. Rev. lett. 96, 256802 (2006).

[24] J.E. Drut and T.A. Lahde, Phys. Rev. B 79, 165425 (2009).

[25] G.Z. Liu, W. Li and G. Cheng, Phys. Rev. B 79, 205429 (2009).

[26] O. V. Gamayun, E. V. Gorbar and V. P. Gusynin, Phys. Rev. B 81, 075429 (2010).

[27] V. N. Kotov, B. Uchoa, V. M. Pereira, A. H. Castro Neto and F. Guinea, arXiv:1012.3484.

[28] Y. Nambu and G.J. Lasinio, Phys. Rev. 122, 345 (1961).

[29] I.J.R. Aitchison, N.Dorey, et al., Phys. Lett. B 294, 91 (1992).

[30] M. He, H.T. Feng, W.M. Sun and H.S. Zong, Mod. Phys. Lett. A 27, 449 (2007).

[31] H.T. Feng, F.Y. Hou, X. He, W.M. Sun and H.S. Zong, Phys. Rev. D 73, 016004 (2006).

[32] H.T. Feng, W.M. Sun, D.K. He and H.S. Zong, Phys. Lett. B. 661, 57 (2008).

[33] H.T. Feng, M. He, W.M. Sun and H.S. Zong, Phys. Lett. B 688, 178 (2010).

[34] A. Ayala, A. Bashir, A. Raya, et al., J. Phys. G 37, 015001 (2010).

[35] I. Affleck and J. B. Marston, Phys. Rev. B 37, 3774 (1988).

[36] L.B. Ioffe and A.I. Larkin, Phys. Rev. B 39, 8988 (1989).

[37] D.H. Kim, P.A. Lee and X.G. Wen, Phys. Rev. Lett. 79, 2109 (1997).

[38] D.H. Kim and P.A. Lee, Ann. Phys. (NY) 272, 130 (1999).

[39] W. Rantner and X.G. Wen, Phys. Rev. B 66, 144501 (2002).

[40] G.Z. Liu and G. Cheng, Phys. Rev. D 67, 065010 (2003).

[41] N. Dorey and N.E. Mavromatos, Nucl. Phys. B 386, 614 (1992).

[42] N. Dorey and N.E. Mavromatos, Phys. Lett. B2 66, 163 (1991).

[43] G.E. Volovik JETP Lett. 58, 469 (1993).

[44] N.E. Hussey, Adv. Phys. 51, 1685 (2002).

[45] S.G. Sharapov and J.P. Carbotte, Phys. Rev. B 73,094519 (2006).

[46] H. Jiang, G.Z. Liu and G. Cheng, Phys. Rev. B 79,174503 (2009).

[47] V.P. Gusynin, V.A. Miransky and I.A. Shovkovy, Phys. Rev. Lett. 73, 3499 (1994).

[48] V.P. Gusynin, V.A. Miransky and I.A. Shovkovy, Phys. Rev. D 52, 4718 (1995).

[49] E.J. Ferrer, V.P. Gusynin and V. de la Incera, Eur. Phys. J. B33, 397 (2003).

[50] E.J. Ferrer and V.de la Incera, Nucl. Phys. B 824, 217 (2010).

[51] A.S. Melnikov, J. Phys. Condens. Matter 21, 4219 (1999).

[52] M. Franz and Z. Tesanovic, Phys. Rev. Lett. 84, 554 (2000). 